\title{
Study on the Supporting Force of Water Spider and its Experimental Model on Water Surface
}

\author{
Wang Qingcheng ${ }^{1, a}$, Yang Xiaodong ${ }^{2, b}$ and Mao Ning ${ }^{3, c}$ \\ 1. School of Mechanical Engineering, Jilin Teachers' Institute of Engineering \\ and Technology, Changchun 130052, China \\ 2. School of Mechanical and Electric Engineering, Changchun Institute of \\ Technology, Changchun 130021, China \\ 3. China North Vehicle Research Institute, Beijing 100072, China

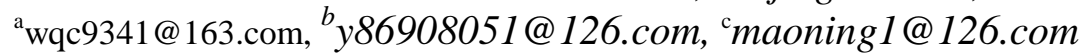

\begin{abstract}
Accuracy of designing and producing a micro-force measuring device is $0.1 \mu N$, through the device, the maximum supporting force of 5 livingwater spiders sample were measured, the maximum supporting force of living water striders is 24.8 times its weight. Water spider has large supporting force on water surface, super-hydrophobic structures of water spider legs play important role. designing and producing 5 water strider models, whose legs were made of aluminum wire with $0.45 \mathrm{~mm}$ diameler, super-hydrophobic processing to surfaces of aluminum wire, its contact angle is 154.5 expeximentally measured that the maximum supporting force of 5 water spider models. Through mechanical analysis, established supporting force mathematical modet of the water spider model on water surface, according to the model calculated the maximum supporting force of water spider model. Compare errors between measured values and calculated values of supporting forces, the ratio of the error value is less than $8.39 \%$, verified the correctness the mathematical model of supporting force.
\end{abstract}

Key words: waterspider, model, supporting force, measuring device, super-hydrophobic

\section{Introduction}

Water spider is a kind of insect living in ponds, lakes and other water areas, which has 8 legs. In recent year, water strider's superior supporting force have drawn the attention of scientists and engineering technicians and become one of the hot topics researched [1-3]. Jiang, et al., [4] deemed that superior supporting force of water strider's legs on water surface is due to its super hydrophobic structure, through experiment they found that the contact angle of water strider's legs is $167^{\circ}$ and the maximum supporting force of water strider's single hind leg is about 15 times of its body weight. Zhang, et al., [5] conducted chemical modification for gold thread surface, and found that the gold thread with diameter of $1 \mathrm{~mm}$ could float on water surface when its contact angle was $150^{\circ}$. Feng, et al., [6] conducted theoretical research on the supporting force of super hydrophobic thin rod floating on water surface, and thought that the supporting force of super hydrophobic surface were related to such factors as its contact angle and contact length.

This paper designed and produced the measurement device of micro-force, measuring supporting force of living water spider and its experimental model. Through mechanical analysis, established the supporting force mathematical model of water spider on water 
surface, calculated the maximum supporting force of water spider on water surface according to the model, compared with the experimental measurements, verified the correctness the mathematical model.

\section{Supporting Force Experimental of Living Water Spider}

\subsection{Design of the Measuring Device}

The structure of self-made measuring device is shown in Figure 1, which was mainly used to measure the maximum supporting force of water strider's legs and maximum dimple depth. In this device, analytical balance (GH-252, accuracy of $0.01 \mathrm{mg}$ ) was made by A\&D Co., Ltd; the graduated scale was made of steel, with an accuracy of $0.1 \mathrm{~mm}$; the guide rail was used to ensure the stability in the process of water strider's leg moving downward to water surface; the feed unit was ball screw nut pair, which was driven by AC servo motor controllea by microcomputer, with an accuracy of $0.001 \mathrm{~mm}$. The entire experiment proces was recorded by CCD. Each group of experimental data was measured for 5 times, with mean value taken.

\section{Figure 1. Structura) Diagram of Measuring Device}

\subsection{Supporting Force Measwing Experiment}

Dozens of water spider had been captured in South Lake Park in Changchun City, 5 water spider samples were Pick out among them. Water spider samples were fixed on the graduated scale of measuring device with double-sided tape, control spider living water strider uniform downward movement, until water strider legs touching the water, pressed the water dimple on water surface, measured the maximum value of supporting force, measurement experimental were shown inFigure 2. The maximum supporting force of 5 water spider sample are shown in Table , the maximum supporting force of 5 water spider sample is 24.8 times its own weight.

Table 1. Supporting Force of Living Water Spider

\begin{tabular}{lccccc}
\hline \multicolumn{1}{c}{ Items } & $\mathrm{S} 1$ & $\mathrm{~S} \mathrm{2}$ & $\mathrm{S} \mathrm{3}$ & $\mathrm{S} \mathrm{4}$ & $\mathrm{S} \mathrm{5}$ \\
\hline Body weight $(\mu \mathrm{N})$ & 207.8 & 259.7 & 339.1 & 404.7 & 497.8 \\
The maximum supporting force $(\mu \mathrm{N})$ & 4421.8 & 6432.7 & 7825.3 & 8149.7 & 8361.2 \\
Coefficient of supporting force & 21.3 & 24.8 & 23.1 & 20.1 & 16.8 \\
\hline
\end{tabular}




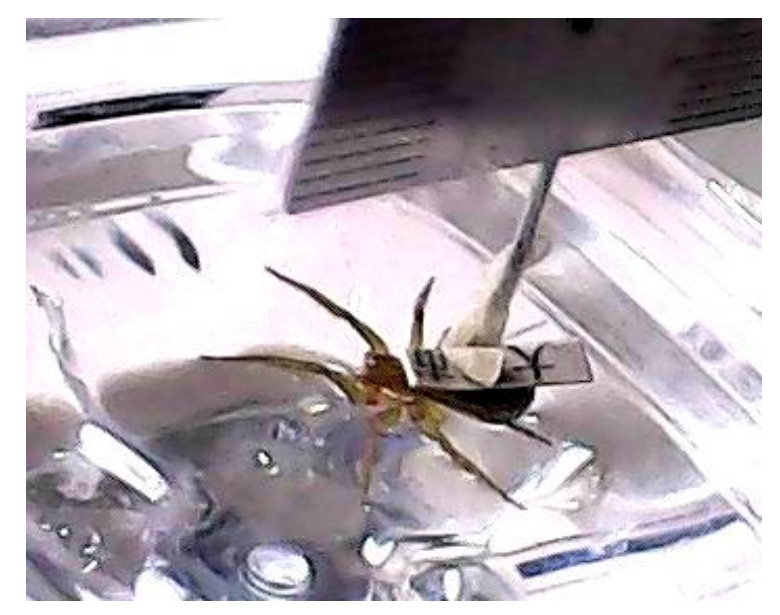

\section{Figure 2. Supporting Force Experimental of Living Water Spider}

\subsection{Microstructure of Water Spider Legs}

Water spider has a large supporting force on water surface, Super-hydrophobicity of water spider legs play an important role, the contact angle of water spider legs was $159.6^{\circ}$ through the contact angle measuring instrument. Microstructure of water spider legs plays a major role, grease play a supporting role for super-hydrophobicity, The legs of water spider sample were cut, fixed on the stage, spraying treatment, observed under the scanning electron microscope (S-300N, HITACHI Company). Water spiden legs covered with micron scale setae, like a feather duster, each setae distributed nanoscale fluff, which formed micro-nano composite structure, which can absorb a lot of air, forming a layer of air film, Prevent water droplets wet, Situation shown in Figure 3.

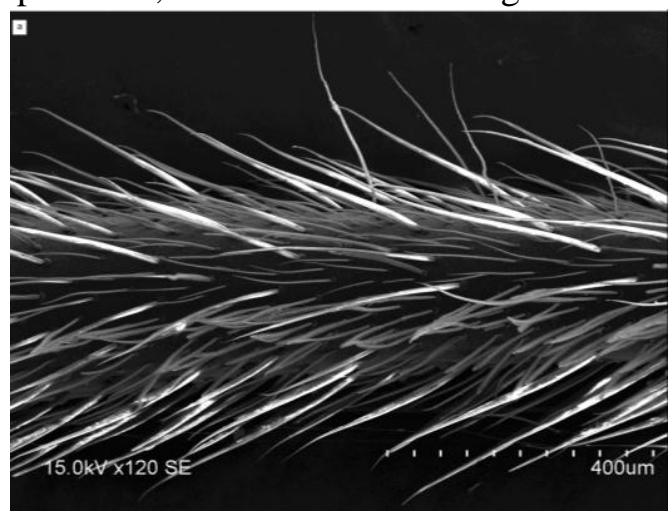

(a) Water spider legs covered with setae

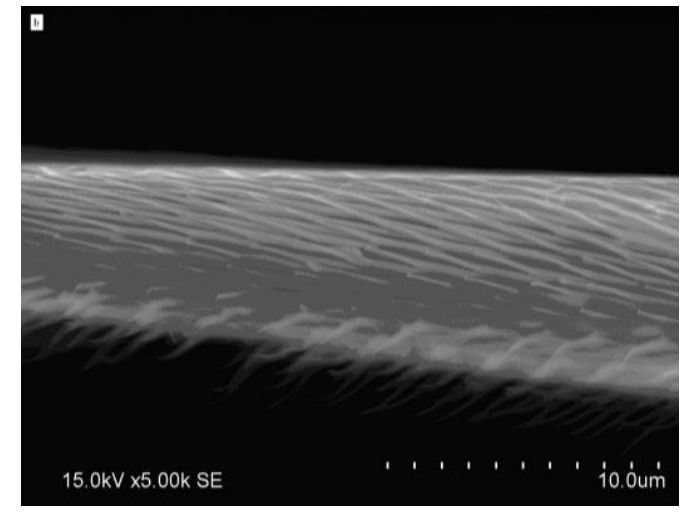

(b) Microstructure of single setae

Figure 3. SEM of Water Spider legs

\section{Supporting Force Measuring and Manufacturing of Water Spider Model}

\subsection{Manufacturing of Water Spider Model}

5 water spider models were fabricated. Their body is made of the steel sheet. The water spider model has 8 legs, which are made of aluminum wire diameter of $0.45 \mathrm{~mm}$ which was super-hydrophobic treatment. Leg length and body weight of water spider model are shown in Table 2, the water spider model is shown in Figure 4.

\section{Table 2. Parameters of Water Spider Model}




\begin{tabular}{lccccc}
\hline \multicolumn{1}{c}{ Model } & M1 & M2 & M3 & M4 & M5 \\
\hline Leg length $(\mathrm{mm})$ & 30 & 35 & 40 & 45 & 50 \\
Body weight $(\mathrm{mN})$ & 5.95 & 6.90 & 7.83 & 8.41 & 9.17 \\
Supporting force $(\mathrm{mN})$ & 36.28 & 42.75 & 49.36 & 53.84 & 59.58 \\
Coefficient of supporting force & 6.1 & 6.2 & 6.3 & 6.4 & 6.5 \\
\hline
\end{tabular}

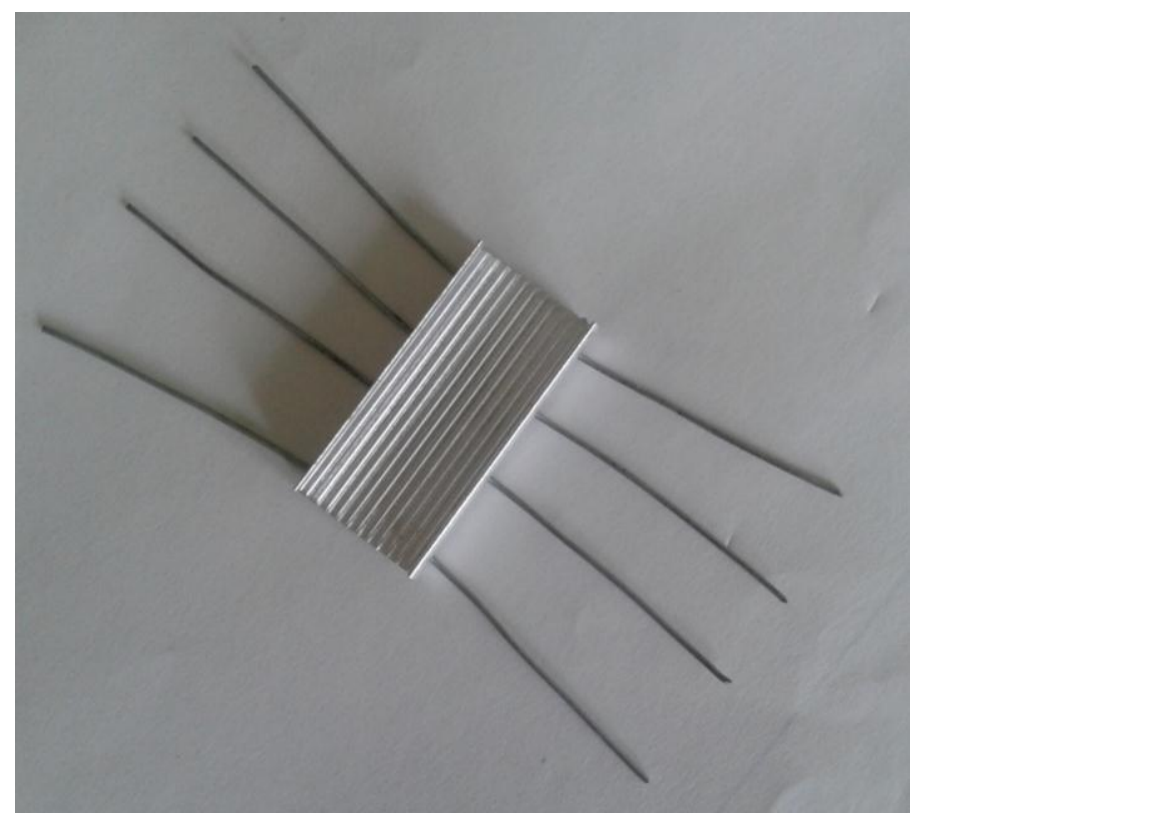

Figure 4. Wate-Spider Model

The aluminum wire used in this experiment was purchased from Shanghai Chemical Reagent Co., Ltd, the test steps are as follows.

(1) Aluminum wire was immersed in acetone solution for $2 \mathrm{~min}$, removing the impurities of the copper surface washed with pure water and dried.

(2) Aluminum wire was immersed into $20 \mathrm{mM}$ silver nitrate solution for 20 seconds, first washed with pure water, then dried with the blast oven (JC101, made by Shanghai Instrument Co., Ltd).

(3) Aluminum wire was observed under SEM (S-300N, made by HITACHI Co., Ltd), a layer of silver bullion was deposited on the copper surface, the shape of silver bullion were similar to the feathers, its length is about $7 \mu \mathrm{m}$, width is about $2 \mu \mathrm{m}$.

(4) Aluminum wire was immersed into the pre-hydrolysis $\mathrm{C} 8 \mathrm{~F} 13 \mathrm{H} 4 \mathrm{Si}(\mathrm{OCH} 2 \mathrm{CH} 3)$ methanol solution whose concentration is $1.0 \mathrm{wt} \%$, soaked for about 1 hour in room temperature, placed in the oven (202-3AB, made by Tianjin Instrument Co., Ltd) and heated for 1 hour at $130^{\circ} \mathrm{C}$. Electron microscopy images after processed super-hydrophobic is shown in Figure 5.

After the above treatment, contact angle of aluminum surface is $154.5^{\circ}$, which was measured by contact angle measurement instrument (DSA100, KRUSS Co., Ltd). 


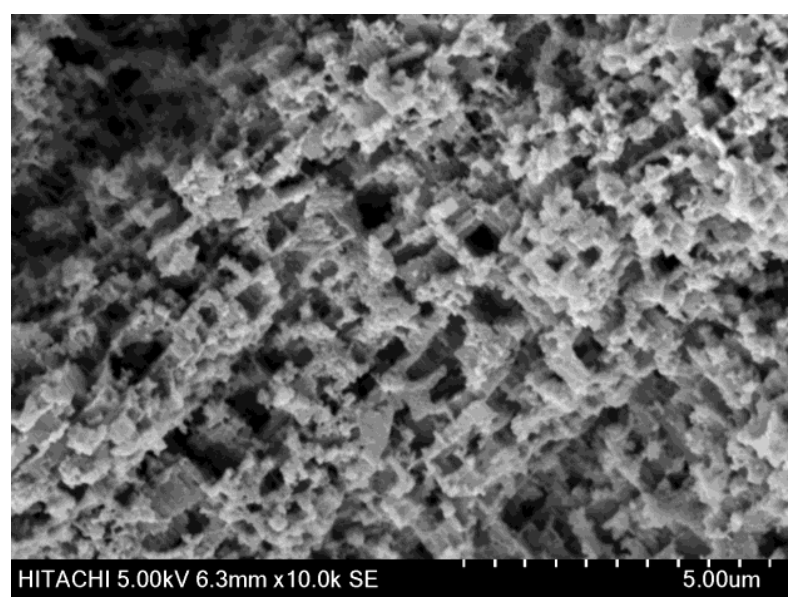

Figure 5. SEM of Super-hydrophobic Aluminum

\subsection{Measuring Supporting Force of Water Spider Model}

The water spider model was placed in a beaker filled with water, which can float on water surface as shown in Figure 4. The sand was added to the water spider model with tweezers until its legs Pierce the water surface. The sand Immersed in water was taken out and dried, the sand quality and water spider model quality is the maximum supporting force of the water spider model, as shown in Table 2.

\subsection{Mechanical Analysis of Water Spider Model}

When water strider stays on water surface calmly, each leg will cause the dimple. Assuming that water strider's leg is cylindrical the forces on its cross-section are as shown in Figure 6.

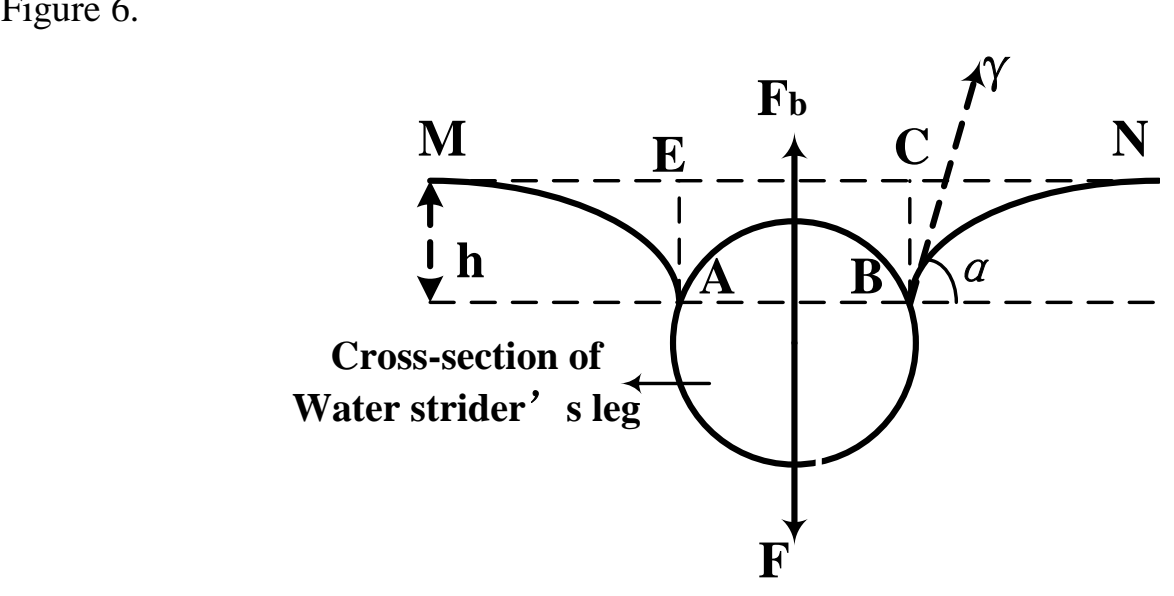

Figure 6. Supporting Force Model of Water Strider's Leg

In Figure 6, the dashed line MN represents the horizontal plane; A and B are the contact points between water strider's leg and water surface. Water strider's legs were subject to three forces on water surface, namely pressure F (including body weight supported by leg and force of gravity of leg), surface tension $\gamma$ and buoyancy force $\mathrm{Fb}$. The buoyancy force is vertically upward, which can be obtained by integrating the hydrostatic pressure over the water strider's legs in contact with water [7-8], and is equal to water displacement of rectangle ABCE drawn 
by dashed lines in Figure 6. Since the diameter of water strider's leg is very small and approximately equal to the length of line segment $\mathrm{AB}$, the expression of buoyancy force $\mathrm{Fb}$ is

$$
F_{b}=\rho D h L g
$$

In Eq.1, $\rho$ represents water density, $g$ represents gravitational acceleration, $\mathrm{h}$ represents dimple depth, D represents the diameter of water strider's leg, L represents the length of water strider's leg. The surface tension is in the tangential direction of Point B on liquid-gas interface, and the expression of its vertical component is

$$
F_{s}=2 L \gamma \sin \alpha
$$

In Eq.2, $\gamma$ represents surface tension constant, $\alpha$ represents horizontal angle of tangent of Point B on liquid-gas interface. The vertical component of surface tension was obtained by integrating the curvature pressure over the water strider's legs in contact with water [7-8], which is equal to the sum of water displacement of AME area and water displacement of $\mathrm{BCN}$ area in Figure 6.

Since the both sides of dimple caused by water strider's leg are symmetrical, the horizontal components of surface tension cancel out each other. In vertical direction, ster strider's leg is subject to the pressure, buoyancy force and vertical component of surface tension; the upward force and the downward force are a pair of baranced force. The supporting force $(\mathrm{F})$ of water strider's leg on water surface is equal to the sum of buoyanicy force and vertical component of surface tension, and its expression is

$$
F=F_{s}+F_{b}=2 L \gamma \sin \alpha+\rho g D h L
$$

Seen from Eq.3, the supporting force of water strider's $\mathrm{s}$ eg is related to such factors as $\mathrm{L}, \mathrm{h}$, $\alpha, \mathrm{D}, \gamma, \rho, \mathrm{g} . \rho, \mathrm{g}$ and $\gamma$ are parameters related to the living environment of the water spider, $\mathrm{L}$ and D are parameters related to wate spider's leg, $h$ and $\alpha$ is parameters related to dimple. The supporting force of water spider on watecsurface is composed of buoyancy force and vertical component of surface tenston, because diameter of water spider's leg is in the grade of micron, the buoyancy forge is only about 1/40 of surface tension [9]. According to the existing research literature 10 , the relational expression of $\alpha$ and $\mathrm{h}$ is

$$
h=\sqrt{\frac{2 \gamma}{\rho g}(1-\cos \alpha)}
$$

When $\alpha=\pi / 2$, supporting force of water strider's leg would reach the maximum value [9], comprehensive analysis expressions (3) and (4) will get expression (5).

$$
F=2 L \gamma+D L \sqrt{2 \gamma p g}
$$

\subsection{Error Analysis of Supporting Force of Calculated Values and Measured Values}

According to Eq.5, because $D=0.45 \mathrm{~mm}, \gamma=72.8 \times 10^{-3} \mathrm{~N} / \mathrm{m}, \rho=10^{3} \mathrm{~kg} / \mathrm{m}^{3}, \mathrm{~g}=9.8 \mathrm{~N} / \mathrm{kg}$, the maximam supporting force of 5 water spider models are calculated, as shown in Table 3 . By the data in Table 3, legs of the water spider model are longer, its supporting force on water surface is greater.

Table 3. The Maximum Supporting Force of 5 Water Spider Models

\begin{tabular}{lccccc}
\hline \multicolumn{1}{c}{ Model } & M1 & M2 & M3 & M4 & M5 \\
Leg length (mm) & 30 & 35 & 40 & 45 & 50 \\
Supporting force $(\mathrm{mN})$ & 39.03 & 45.53 & 52.04 & 58.54 & 65.04 \\
\hline
\end{tabular}


According to Table 2 and Table 3, comparative analysis of errors between measured values and calculated values of supporting forces was conducted, as is shown in Figure 7. The error value and the ratio of the error value of supporting forces are shown in Table 4.

Table 4. Error Value and the Ratio of the Error Value of Supporting Forces

\begin{tabular}{lccccc}
\hline \multicolumn{1}{c}{ Model } & M1 & M2 & M3 & M4 & M5 \\
Error value $(\mathrm{mN})$ & 2.75 & 2.78 & 3.68 & 4.68 & 5.46 \\
Ratio of the error value 2(\%) & 7.05 & 6.10 & 7.07 & 7.99 & 8.39 \\
\hline
\end{tabular}

The measured value is less than the calculated value of supporting forces of the water spider model, which has two reasons.

(1) When supporting forces of the water spider model was measuring, the actual the maximum depth of the dimple is possible smaller than the theoretical calculation.

(2) It is difficult to maintain a state of equilibrium for the water spider model, the process of which the sand was add to the model, because transient partial load, The modei has not yet reached maximum value, water surface was pierced by its legs.

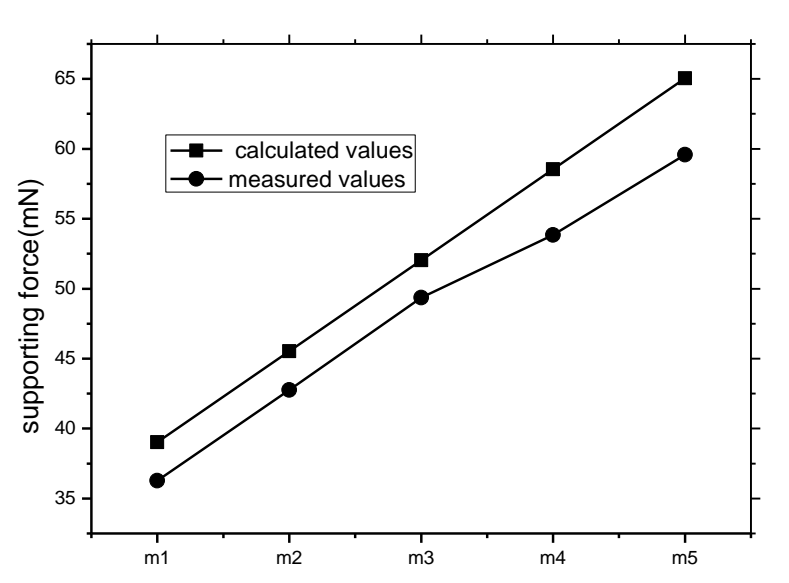

Figure 7 . Error Analysis Calculated Values and Measured Values

\section{Comparative Analysis of Supporting Force of water spider and its model}

Comparative analys supporting force coefficient of water spiders and experimental model, coefficient of supporting force of water spider is shown in Table 1, coefficient of supporting force of water spider model is shown in Table 2. It can be seen that supporting force coefficient of water spider is greater than its experimental model. In addition to the super hydrophobic factors, there may be other unknown factors, it needs further research.

\section{Conclusion}

(1) The self-made measuring device was used in the experiment to measure the maximum dimple depth and maximum supporting force of water spider's leg on water surface.

(2) The comparative analysis of measured values and calculated values of supporting forces of water spider's legs was conducted, with error rate lower than $8.39 \%$.

(3) The research results laid theoretical and experimental foundation for further revealing the superior supporting mechanism of water spider and its experimental model on water surface. 


\section{Acknowledgements}

This work was supported by National Natural Science Foundation of China (51275055), Jilin Province Science and Technology Development Project (20140101058JC) and Jilin Provincial Department of Education funded project (2013370).

\section{References}

[1]. M. Dickinson, “Animal locomotion: how to walk on water", Nature, vol. 424, no. 6, (2003), pp. 621-622.

[2]. D. L. Hu, B. Chan and J. W. M. Bush, "The hydrodynamics of water strider locomotion”, Nature, vol. 424, no. 11, (2003), pp. 663-666.

[3]. P. J. Wei, Y. X. Shen and J. F. Lin, "Characteristics of Water Strider Legs in Hydrodynamic Situations", Langmuir, vol. 25, no. 12, (2009), pp. 7006-7009.

[4]. G. Xuefeng and J. Lei, "Water-repellent legs of water striders", Nature, vol. 432, no. 8, (2004), pp. 26-26. *

[5]. S. Feng, N. Jia, L. Jianlin, et al., "Towards understanding why a super hydrophobic coating is needed by water striders", Adv. Mater, vol. 19, (2007), pp. 2257-2261.

[6]. L. Jianlin, F. Xiqiao and W. Gangfeng, "Buoyant force and sinking conditions of a hy drophobic thin rod floating on water", Physical Review E, vol. 76, (2007), pp. 066103.

[7]. D. Vella, D.-G. Lee and H.-Y. Kim, "The Load Supported by SmalNFloating Objects", Langmuir, vol. 22, (2006), pp. 5979-5981.

[8]. P. Qinmin, L. Jia and Z. Qing, "A water strider-like model with large and stable loading capacity fabricated from super hydrophobic copper foils", Applied Materials \& Interfaces, vol 2, no.7,(2010), pp. 2026-2030.

[9]. W. Qingcheng, Y. Xiaodong, et al., "Modeling and analysis of the supporting force of water strider's legs", Applied Mechanics And Mechanical, vol. 138, (2011), pp. 356-361.

[10].W. Shuhui and W. Licheng, "Statics analysis on the leg of water strider robot", Journal of Beijing University of Aeronautis and Astronautis, vol. 36, no. 10, (2010), pp. 1176-1179.

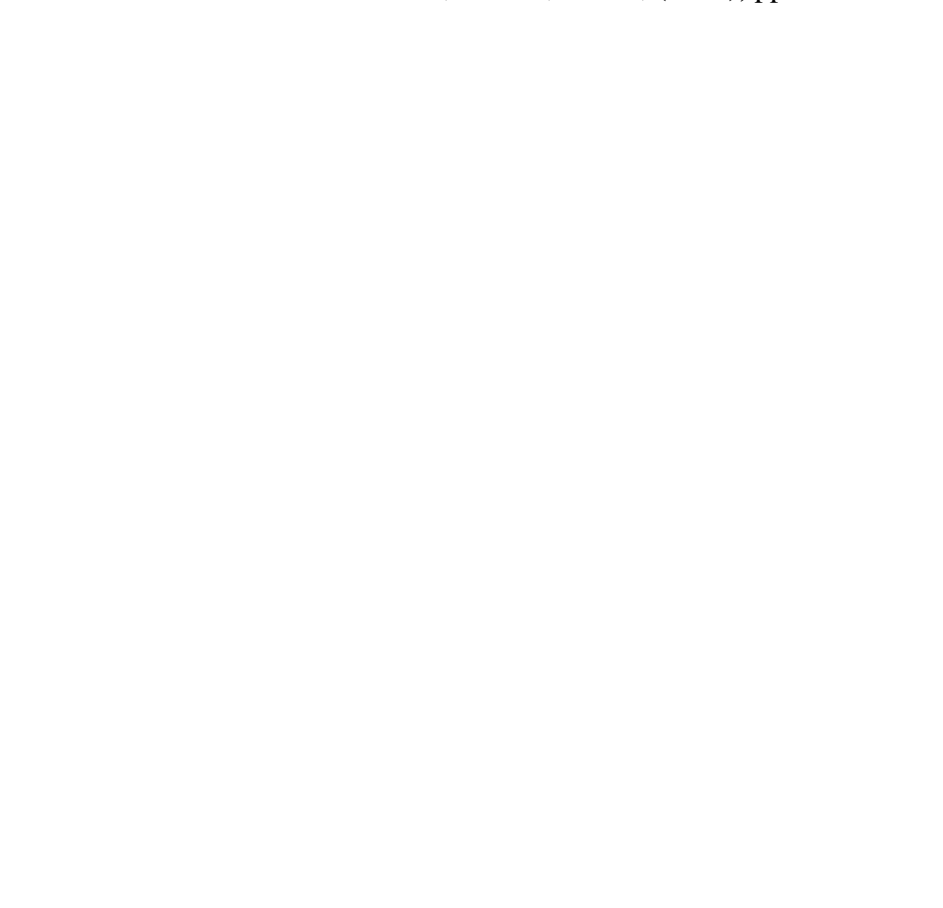

